\title{
Dermatological manifestations in the intensive care unit - A prospective study
}

\section{Heena Gupta', Mrinal Gupta ${ }^{2}$}

\author{
${ }^{1}$ Governement Medical College, Jammu, India, ${ }^{2}$ Consultant Dermatologist, Treatwell Skin Centre, Jammu, India
}

Corresponding author: Dr. Mrinal Gupta, E-mail: drmrinalgupta@yahoo.com

\begin{abstract}
Background: Intensive care unit is a specialized controlled unit where the critically ill patient is dependent on the caregivers. Aims: This study was carried out to study the dermatological manifestations in patients admitted in intensive care unit. Materials and Methods: It was a prospective study carried out over a period of six months where the patients admitted in the intensive care unit having some dermatological manifestations were examined and the findings were noted. Results: A total of 273 patients were examined (M: F 154:119) were examined out of which 50 patients (18.31\%) (M: F 31:19) were having some dermatological manifestations. The age range of patients ranged from 3 years to 84 years with a mean age of 46.356 .43 years. The stay in ICU varied from 3-43 days with a mean of 17.15 days. Infectious diseases constituted $52 \%(\mathrm{n}=26)$ of the dermatological conditions with the most common being fungal infections $(26 \%, n=13)$ and bacterial infections $(16 \%, n=8)$. Among the non-infectious dermatoses, the most common were drug reactions $(24 \%, n=12)$, followed by friction blisters $(12 \%, \mathrm{n}=6)$ and dermatitis $(4 \%, \mathrm{n}=2)$. Conclusions: Dermatological diseases are common in patients in intensive care unit which can have an impact on the duration of stay, alteration in therapy and mortality among the patients.
\end{abstract}

Key words: Dermatological disorders, Comorbidity, Drug reactions, Intensive care unit

\section{INTRODUCTION}

Intensive care unit (ICU) is a specialized unit to manage critically ill patients. As the patient is already on life support, on multiple medications and immobile, it creates an environment for growth of organisms which can lead to a variety of nosocomial infections including cutaneous infections $[1,2]$. Moreover, as the mobility of the patient is impaired, the chances of complications like decubitus ulcers are increased manifold. Various dermatological problems may develop in ICU patients as a result of primary pathologies, their complications, and complex treatment regimens used for therapy. Dermatological conditions can increase the risk of complications, increase in the stay duration, can lead to alteration in the treatment regimens and can also increase the morbidity and mortality [3,4].

We undertook this study to study the dermatological manifestations in the patients admitted in the intensive care unit.

\section{MATERIALS AND METHODS}

It was a prospective study carried out over a period of six months in which all the patients admitted in the intensive care unit were examined for the presence of any dermatological condition and the findings were recorded in a predesigned proforma by a dermatologist. Age, sex, comorbid conditions, dermatological disorders, tissue culture results, time to consultation, duration of ICU stay were recorded among patients admitted to ICU.

\section{RESULTS}

A total of 273 patients were examined (M: F 154:119) were examined out of which 50 patients (18.31\%) (M:F 31:19) were having some dermatological manifestations. The age range of patients ranged from 3 years to 84 years with a mean age of 46.356 .43 years. The stay in ICU varied from 3-43 days with a mean of 17.15 days. 
Among the dermatological conditions, infectious diseases constituted $52 \%(n=26)$ of the total disease load. Fungal infections were the most common infectious diseases, constituting $26 \%(n=13)$ of the total disease with dermatophytoses $(n=7)$ being the commonest in the form of tinea cruris $(n=3)$, tinea pedis and manuum $(n=2)$ and onychomycosis $(n=2)$ followed by Candidiasis $(n=6)$ in the form of oral thrush $(n=4)$ and balanoposthitis and vulvovaginitis $(n=1$ each). Bacterial infections constituted $16 \%$ $(n=8)$ of the infectious conditions which included wound infections $(n=4)$, furunculosis and carbuncles in $4 \%(n=2)$ erysipelas and cellulitis in 25 each $(n=1)$. Among the non-infectious dermatoses, the most common were drug reactions $(24 \%, \mathrm{n}=12)$ in the form of maculopapular drug rash in $14 \%(n=7)$, acneiform eruption in $45(\mathrm{n}=2)$, Stevens Johnson's syndrome and Toxic epidermal necrolysis in $4 \%(n=2)$ and fixed drug eruption in $2 \%(\mathrm{n}=1)$. It was followed by friction blisters $(12 \%, n=6)$ and dermatitis $(4 \%$, $\mathrm{n}=2)$. Urticaria, psoriasis and vasculitis were seen in one patient each.

\section{DISCUSSION}

In an intensive care unit, prolonged immobilization, malnutrition, impaired tissue perfusion, immune system dysfunction, fluctuations in body temperature, inadequate hygiene, hyperpyrexia, medications, and skin injuries may cause the disruption of the skin barrier function, which predispose the patients to a large number of dermatological disorders ranging from infections to non infectious dermatoses like drug reactions and frictional blisters [1-4]. The incidence of dermatological disorders in patients in ICU has been reported to vary from $2.2 \%$ to $21.5 \%[3,5]$. In agreement with the literature, our study demonstrated an incidence of $18.31 \%$.

Skin infections constitute the major dermatological manifestations in the ICU. Fischer et al reported an incidence of $29 \%$ of different skin infections whereas Emre et al reported an incidence of $38.9 \%$ [2,5]. Among the infections, fungal infections are the most common infections with Candida being the most commonly implicated pathogen, which is attributed to increased humidity, administration of broad spectrum antibiotics, parenteral nutrition, immunosuppressive agents, and comorbidities [2,6]. In our study, the incidence of infective skin disorders was $52 \%$, among which fungal infections were the most common with dermatophytoses constituting $14 \%$ of the total infection load, followed by Candidiasis in $12 \%$. The low incidence of bacterial infections could be attributed to the co administration of antibiotics to the patients.

Drug reactions constitute another major dermatological manifestation in the ICU with various studies reporting an incidence varying from $9.3 \%$ to $21.6 \%[2-5,7]$. Emre et al reported that drug reactions occurred in $14.5 \%$ of patients staying at intensive care units with maculopapular rash being the most common subtype [2]. Antibiotics and chemotherapeutic agents are the most common drugs implicated in causing drug reactions. Anticonvulsive agents, allopurinol, diuretics, and non-steroidal antiinflammatory drugs may also cause drug reactions [7]. In our study, drug reactions constituted $24 \%$ of all dermatological manifestations and maculopapular drug reactions were the most common type.

Apart from the drug reactions, other non infectious dermatoses like friction blisters, vasculitis, seborrheic dermatitis are also very common in patients in ICU. Emre et al reported that dermatoses are observed at intensive care units at a rate of $46.6 \%$, and the most common pathologies were frictional bullae and allergic contact dermatitis, whereas, Fischer et al, reported an incidence of 49.4\% [2,5]. Another study reported that the most common skin disorders were stasis dermatitis $(25 \%)$ and diaper dermatitis (25\%) in medically treated critically ill patients [8]. In our study, apart from drug reactions, the most common dermatoses were friction blisters $(12 \%, \mathrm{n}=6)$ and dermatitis $(4 \%, \mathrm{n}=2)$. Urticaria, psoriasis and vasculitis were seen in one patient each. Immobilization, numerous medications, higher age increase the fragility of skin, therefore when frequent friction is applied, it leads to friction blisters. Moreover, continuous use of topical irritant agents like electrodes, chlorhexidine also predispose to the development of eczemas in the already fragile skin $[9,10]$.

Skin diseases can markedly prolong the stay in ICU. Many factors including age, multiple comorbid conditions, duration of ICU stay, time to consultation, and mortality contribute to the development of dermatological disorders. Increased cooperation is needed among ICU physicians and dermatology department to reduce the dermatological complications, increase the quality of patient care, shorten hospital stay, and reduce mortality rate. 


\section{STATEMENT OF HUMAN AND ANIMAL RIGHTS}

All procedures followed were in accordance with the ethical standards of the responsible committee on human experimentation (institutional and national) and with the Helsinki Declaration of 1975, as revised in 2008.

\section{STATEMENT OF INFORMED CONSENT}

Informed consent was obtained from all patients for being included in the study.

\section{REFERENCES}

1. Dunnill M, Handfield-Jones S, Treacher D, McGibbon D. Dermatology in the intensive care unit. Br J Dermatol. 1995;132:226-35.

2. Emre S, Emre C, Akoglu G, Demirseren DD and Metin A. Evaluation of dermatological consultations of patients treated in intensive care unit. Dermatology. 2013;226:75-80.

3. Badia M, Serviá L, Casanova JM, Montserrat N, Vilanova J, Vicario E, et al. Classification of dermatological disorders in critical care patients: a prospective observational study. J Crit Care. 2013;28:220, e1-8.

4. Wollina U, Nowak A. Dermatology in the intensive care unit. Our Dermatol Online. 2012;3:298-303.

5. Fischer M, Soukup J, Wohlrab J, Radke J, Marsch W. Key dermatological symptoms in the intensive care unit. Int J Dermatol. 2004;43:780-2.

6. Acar A, Oncül O, Kuecuekardali Y, Ozyurt M, Haznedaroğlu T, Cavuşlu S. Epidemiological features of candida infections detected in intensive care units and risk factors affecting mortality. Mikrobiyol Bul. 2008;42:451-61.

7. Fischer M, William T, Wohlrab J. Skin diseases in intensive care medicine. J Dtsch Dermatol Ges. 2009;7:108-15.

8. Kara A, Ortaç E, Hapa A, Öcal S, Topeli A. Yoğun bakım ünitesinde karşılaşılan dermatolojik sorunlar ve dermatoloji konsültasyonu. Turkish J Med Surg Int Care Med. 2015;6:1.

9. Del Mar Campos-Fernández M, Ponce-de- León-Rosales S, Archer-Dubon C, Orozco- Topete R. Incidence and risk factors for cutaneous adverse drug reactions in an intensive care unit. Rev Invest Clin. 2005;57:770-4.

10. Liippo J, Kousa P, Lammintausta $\mathrm{K}$. The relevance of chlorhexidine contact allergy. Contact Dermatitis. 2011;64:229-34.

Copyright by Heena Gupta, et al. This is an open-access article distributed under the terms of the Creative Commons Attribution License, which permits unrestricted use, distribution, and reproduction in any medium, provided the original author and source are credited.

Source of Support: Nil, Conflict of Interest: None declared. 